\title{
Comparison of Extubation Outcomes Following T-piece Trial versus Pressure Support/Continous Positive Airway Pressure in Postsurgical Patients
}

\author{
Lakshmi Kumar, A. R. Meghalakshmi, Anu Vasudevan, Sunil Rajan, Jerry Paul \\ Department of Anesthesia and Critical Care, Amrita Institute of Medical Sciences and Research Centre, Kochi, Kerala, India
}

Abstract

Background: Pressure support with continuous positive airway pressure (CPAP) and T-piece trials are the most common spontaneous breathing trial used to test readiness for extubation. Aim: We aimed to compare extubation failures defined by the need for reintubation within $48 \mathrm{~h}$ following T-piece trial versus extubation directly from pressure support (PS) ventilation in postoperative patients. Patients and Methods: This was a prospective cross-sectional study conducted in the postsurgical patients. Hemodynamic parameters and respiratory variables were measured before and after weaning trials. Outcomes after extubation, need for noninvasive-assist ventilation following extubation, duration of oxygen therapy, and time of shifting from the Intensive Care Unit (ICU) were also recorded. Results: Fifty patients needed mechanical ventilation postoperatively were recruited for the study. No significant differences were seen in the rate of extubation failures between PS- and T-piece groups. Rapid shallow breathing index recorded at the start of weaning was significantly higher in the T-piece group $(P<0.001)$. The respiratory rate $(\mathrm{RR})$ and heart rate $(\mathrm{HR})$ were significantly higher $(P<0.001)$ and saturation lower $(P=0.035)$ in the group on T-piece trial. The need for respiratory assist devices, oxygenation index, length of ICU stay, duration of oxygen therapy, and mortality were comparable between the two groups. Conclusions: Outcomes of weaning are similar between T piece and CPAP/PS in patients undergoing postoperative mechanical ventilation. Weaning on T piece is associated with higher RR, HR, rapid shallow breathing, and lower saturation than weaning from CPAP/PS but does not affect the length of ICU stay, need for oxygen therapy, or mortality.

Keywords: Extubation, pressure support-continuous positive airway pressure, T-piece trials

\section{INTRODUCTION}

The need for postoperative ventilation in surgical patients represents a unique subgroup of patients in the Intensive Care Unit (ICU). Postoperatively, patients are usually extubated in the operating room unless associated with difficult intubation, unstable hemodynamics, fluid overload, edematous airway, hypothermia, or inadequate recovery from sedatives and muscle relaxants.

Weaning of patients after a period of ventilation is the challenge to intensivists and respiratory care personnel and initiating a spontaneous breathing trial (SBT) is the simplest intervention to test readiness for extubation. ${ }^{[1,2]}$ The most commonly used interventions are SBT on a T piece or on a combination of pressure support (PS) and continuous positive airway pressure (CPAP).

\begin{tabular}{|l|l|}
\hline \multicolumn{2}{|c|}{ Access this article online } \\
\hline Quick Response Code: & Website: \\
\hline
\end{tabular}

PS ventilation is a spontaneous mode of assisted mechanical ventilation where a preset level of pressure generated by the ventilator assists each of the inspiratory efforts. PS is reduced in steps of 2-4 cm $\mathrm{H}_{2} \mathrm{O}$ at a frequency commensurate to the patients' tolerance. The abrupt discontinuation of mechanical ventilation and allowing spontaneous breathing through a $\mathrm{T}$ piece is another simple and most commonly used SBT. Recent studies have shown that a T-piece SBT lasting $>1 \mathrm{~h}$ is a useful test in selecting patients who are ready for extubation. ${ }^{[3,4]}$

Address for correspondence: Dr. Lakshmi Kumar, Department of Anesthesia and Critical Care, Amrita Institute of Medical Sciences and Research Centre, Kochi - 682 024, Kerala, India. E-mail: lakshmi.k.238@gmail.com

This is an open access article distributed under the terms of the Creative Commons Attribution-NonCommercial-ShareAlike 3.0 License, which allows others to remix, tweak, and build upon the work non-commercially, as long as the author is credited and the new creations are licensed under the identical terms.

For reprints contact: reprints@medknow.com

How to cite this article: Kumar L, Meghalakshmi AR, Vasudevan A, Rajan S, Paul J. Comparison of extubation outcomes following T-piece trial versus pressure support/continuous positive airway pressure in postsurgical patients. Indian J Respir Care 2018;7:37-41. 
We aimed to compare the respiratory outcomes of extubation in two groups of ventilated patients assigned to an SBT with either T-piece or PS of $\leq 8 \mathrm{~cm} \mathrm{H}_{2} \mathrm{O}$. The primary objective was to compare extubation failures defined by the need for reintubation within $48 \mathrm{~h}$ following weaning by either method. The secondary objectives included the measurement of weaning index, rapid shallow breathing index (RSBI) at the beginning, $30 \mathrm{~min}$ and $1 \mathrm{~h}$ after weaning initiation, duration of oxygen therapy, length of ICU stay, and mortality.

\section{Patients and Methods}

After obtaining clearance from the local Ethics Committee, this study was conducted in the gastrointestinal, orthopedic, and neurological surgical units of AIMS from December 2016 to March 2017. Fifty patients who required mechanical ventilation after their surgical procedure (abdominal and neurosurgery) for $>12 \mathrm{~h}$ before initiation of weaning were included in this study.

Patients were enrolled in the study only when they fulfilled criteria, namely, Glasgow coma score $>9$, normothermic with temperature below $38.5^{\circ} \mathrm{C}$, a hemoglobin level $>8 \mathrm{~g} / \mathrm{dl}$, normal electrolytes, and on no or minimal doses of vasoactive agents. In addition, the approval of the attending physician on weaning was obtained.

An SBT was initiated when at least 3 of the following criteria were fulfilled, namely,

1. Maximal inspiratory pressure $\leq-25 \mathrm{~cm} \mathrm{H}_{2} \mathrm{O}$

2. Breathing frequency $\leq 35$ breaths $/ \mathrm{min}$

3. Vital capacity $\geq 10 \mathrm{ml} / \mathrm{kg}$ of body weight

4. Arterial oxygen saturation $>90 \%$ for an inspired oxygen fraction of $40 \%$.

Patients were randomly allotted to one of the two groups by computer-generated sequence of random numbers.

- Group I: Patients were directly extubated from a combined PS $<8 \mathrm{~cm} \mathrm{H}_{2} \mathrm{O}$ and PEEP $\leq 5 \mathrm{~cm} \mathrm{H}_{2} \mathrm{O}$. Patients were considered fit for extubation if they tolerated PS-CPAP mode for $>60 \mathrm{~min}$

- Group II: Patients were extubated after a trial on a T-piece for $>30$ min after standard weaning from PS. In this group, SBT was administered with a T-piece, and the patients were monitored continuously for the first $5 \mathrm{~min}$ and then during $30 \mathrm{~min}$ intervals of the trial. Patients who tolerated the SBT for $1 \mathrm{~h}$ or more were considered fit for extubation.

The weaning was continued unless the patient manifested any of the following signs or symptoms suggestive of weaning intolerance

1. Respiratory rate $(\mathrm{RR})>35$ breaths $/ \mathrm{min}$

2. Arterial oxygen saturation $<90 \%$

3. Heart rate $(\mathrm{HR})>140$ beats/min or a sustained increase or decrease in the HR of $>20 \%$ from baseline

4. Systolic blood pressure $>200$ or $<80 \mathrm{mmHg}$

5. Agitation, diaphoresis, or anxiety anytime during the trial.
If the patient manifested any of the above, the weaning trial was aborted, and assist-control ventilation was reinstituted. Patients failing the first SBT were reassessed later after the correction of possible contributory factors and the parameters were recorded.

Data were collected using a pro forma sheet that was filled by the respiratory therapist assigned to the ICU and managing the weaning for the patient [Table 1]. Duration of postoperative mechanical ventilation and requirement of reintubation was documented, and the length of ICU stay and incidence of death were recorded.

Parameters, such as RR and tidal volume $\left(\mathrm{V}_{\mathrm{T}}\right)$, were recorded directly from the ventilator in case of Group I, and minute ventilation and RSBI were calculated accordingly.

In Group II, the ventilator was set on "flow-by" with PS and PEEP both set at zero, and three consecutive values of RR and $\mathrm{V}_{\mathrm{T}}$ were taken, and the average was calculated to get standardized values followed by calculation of expired minute ventilation (MVe) and RSBI. These parameters were recorded at the beginning, $30 \mathrm{~min}$ and at the end of trial. Hemodynamic parameters were recorded at 30 min intervals during the SBT. An arterial blood gas (ABG) was drawn at the beginning, $30 \mathrm{~min}$ and at the end of SBT and oxygenation was assessed by recording $\mathrm{PaO}_{2} / \mathrm{FiO}_{2}$. Postextubation and $24 \mathrm{~h} \mathrm{ABG}$, and hemodynamic parameters were also noted on the following days.

Statistical analysis was done using IBM SPSS 20 (SPSS Inc, Chicago, USA). For all the continuous variables, the results were obtained as in mean \pm standard deviation for categorical variables as percentage. To compare the mean difference of numerical variables between groups, independent sample $t$-test was applied. To obtain the association between two variables, Chi-square test was applied. $P<0.05$ was considered statistically significant.

\section{RESULTS}

Fifty postsurgical patients were randomized to undergo SBT through PS-CPAP and the remaining 25 patients to undergo a T-piece trial after standard weaning from PS-CPAP. The two groups were comparable in terms of demographic profiles and types of surgery.

The demographic variables and duration of mechanical ventilation before extubation trials were comparable between the two groups. The numbers of patients undergoing abdominal surgery were higher in Group II in comparison with Group I [Table 2]. The need for reintubation within $48 \mathrm{~h}$ following extubation was comparable in both groups [Table 2].

The RSBI and the RR were significantly higher in Group II at the start of weaning, $30 \mathrm{~min}$ and $60 \mathrm{~min}$ of the weaning trial $(P<0.05)$. HR was higher in Group II at the start of the trial but comparable at 30 and $60 \mathrm{~min}$, while $\mathrm{MV}_{\mathrm{e}}$ and the $\mathrm{P} / \mathrm{F}$ ratio were comparable between the two groups [Table 3]. The need for noninvasive ventilation (NIV) following extubation and 


\section{Table 1: Proforma for extubation outcomes}

\begin{tabular}{|c|c|c|c|c|}
\hline Name: & $\begin{array}{l}\text { MRD: } \\
\text { Weight (kg): }\end{array}$ & Age: & Sex: & Height $(\mathrm{cm})$ : \\
\hline \multicolumn{5}{|l|}{ Diagnosis: } \\
\hline \multicolumn{5}{|l|}{ Surgical procedure: } \\
\hline \multicolumn{5}{|l|}{ Comorbidities: } \\
\hline \multicolumn{5}{|l|}{ Date of surgery: } \\
\hline ASA grade: & $\mathrm{I} / \mathrm{II} / \mathrm{III}$ & & & \\
\hline Preoperative mechanical ventilation: & Yes/no & & & \\
\hline \multicolumn{5}{|l|}{ Indication of postoperative MV: } \\
\hline \multicolumn{5}{|l|}{ Duration of postoperative MV: } \\
\hline Initial ventilator settings: & $\begin{array}{l}\text { Mode: } \\
\text { PEEP: }\end{array}$ & $\begin{array}{l}\mathrm{FiO}_{2}: \\
\text { PC: }\end{array}$ & $\begin{array}{l}\text { RR: } \\
\text { VTe }\end{array}$ & I:E: \\
\hline Type of SBT: & PS-CPAP/T-piece & & & \\
\hline Adequate respiratory muscle strength: & Yes/no & & & \\
\hline Adequate cough reflex: & Yes/no & & & \\
\hline Easily managed secretions: & Yes/no & & & \\
\hline
\end{tabular}

GCS:

Inotropic supports:

CXR:

RR

$\mathrm{VTe}$

Weaning parameters

0 min:

30 min:

60 min:

RR HR

Hemodynamic parameters

Preextubation $0 \mathrm{~min}$ :

$30 \mathrm{~min}:$

$60 \mathrm{~min}:$

Postextubation:

After $24 \mathrm{~h}$ :

Preextubation 0 min:
30 min:
60 min:

Arterial blood gas values

$\begin{array}{llll}\mathrm{PaO}_{2} & \mathrm{HCO}_{3}^{-} & \text {Lactate } & \mathrm{PaO}_{2} / \mathrm{FiO}_{2}\end{array}$

Postextubation:

After $24 \mathrm{~h}$ :

reintubation were comparable between both groups. There were no differences between the two groups in mortality, duration of oxygen therapy, and length of ICU stay [Table 4].

\section{Discussion}

The primary objective of this study was to evaluate the superiority of weaning using $\mathrm{T}$ piece versus direct extubation from PS. We compared extubation failure defined by the requirement of reintubation within $48 \mathrm{~h}$. This was similar to the rates of reintubation between PS and T-piece in other studies. ${ }^{[1,4,5]}$ The primary indications for reintubation in our study group were respiratory failure and poor level of consciousness. Upper airway obstruction was also found to be a factor in one patient. We did not have patients in the postsurgical group requiring intubation for cardiac failure or aspiration unlike other studies. ${ }^{[6]}$ Although $\mathrm{T}$ piece and PS-CPAP are standard weaning modalities, Haberthür et al. compared weaning on $\mathrm{T}$ piece and PS in comparison with automatic tube compensation (ATC) and concluded that some patients who had failed weaning on $\mathrm{T}$ piece or PS could be successfully extubated on ATC. ${ }^{[7]}$

There is still a lack of consensus on the most effective index or volume for prediction of successful weaning. Conti et al. in a prospective study evaluated 93 patients on mechanical ventilation in an ICU. In a measurement of lung volumes, airway occlusion pressures and RR, they concluded that these 
Kumar, et al.: Extubation outcome - T-piece trial vs PSV

\begin{tabular}{lccc}
\hline \multicolumn{4}{l}{ Table 2: Demographics and reintubation } \\
\hline & $\begin{array}{l}\text { Group I } \\
(\boldsymbol{n}=\mathbf{2 5})\end{array}$ & $\begin{array}{c}\text { Group II } \\
(\boldsymbol{n}=\mathbf{2 5})\end{array}$ & $\boldsymbol{P}$ \\
\hline $\begin{array}{l}\text { Mean age (years) } \\
\text { Gender, } n \text { (\%) }\end{array}$ & $54 \pm 11$ & $51 \pm 14$ & 0.615 \\
$\quad$ Male & $18(72)$ & $15(60)$ & 0.375 \\
Female & $7(28)$ & $10(40)$ & \\
Types of surgery, $n$ (\%) & & & \\
Gastrosurgery & $16(64)$ & $24(96)$ & 0.013 \\
$\quad$ Neurosurgery & $9(36)$ & $1(4)$ & \\
$\begin{array}{l}\text { Duration of postoperative } \\
\text { mechanical ventilation (h) }\end{array}$ & $19.60 \pm 15.75$ & $20.96 \pm 15.31$ & 0.758 \\
Reintubation, $n$ (\%) & & & \\
$\quad$ Yes & $4(16)$ & $3(12)$ & 0.687 \\
No & $21(84)$ & $22(88)$ & \\
\hline
\end{tabular}

\begin{tabular}{lcccc}
\hline \multicolumn{5}{l}{ Table 3: Respiratory outcomes at weaning } \\
\hline Parameter & Time & Group I & Group II & $\boldsymbol{P}$ \\
\hline RSBI & $0 \mathrm{~min}$ & $32 \pm 12$ & $58 \pm 26$ & $<0.001$ \\
& $30 \mathrm{~min}$ & $33 \pm 11$ & $51 \pm 24$ & 0.002 \\
& $60 \mathrm{~min}$ & $32 \pm 12$ & $57 \pm 21$ & 0.001 \\
& $0 \mathrm{~min}$ & $8 \pm 2.6$ & $8.4 \pm 3.1$ & 0.661 \\
$\mathrm{MV}_{\mathrm{e}}$ (L/min) & $30 \mathrm{~min}$ & $7.8 \pm 1.9$ & $8.1 \pm 2.6$ & 0.758 \\
& $60 \mathrm{~min}$ & $8.0 \pm 2.1$ & $8.4 \pm 2.5$ & 0.589 \\
& $0 \mathrm{~min}$ & $402 \pm 107$ & $416 \pm 125$ & 0.667 \\
P/F ratio & $30 \mathrm{~min}$ & $414 \pm 111$ & $459 \pm 128$ & 0.189 \\
& $60 \mathrm{~min}$ & $400 \pm 106$ & $472 \pm 151$ & 0.058 \\
& $0 \mathrm{~min}$ & $16 \pm 5$ & $21 \pm 5$ & 0.001 \\
Respiratory rate (breaths/min) & $30 \mathrm{~min}$ & $16 \pm 4$ & $19 \pm 4$ & 0.002 \\
& $60 \mathrm{~min}$ & $16 \pm 3$ & $21 \pm 4$ & $<0.001$ \\
& $0 \mathrm{~min}$ & $82 \pm 23$ & $97 \pm 19$ & 0.019 \\
Heart rate (beats/min) & $30 \mathrm{~min}$ & $85 \pm 20$ & $96 \pm 21$ & 0.066 \\
& $60 \mathrm{~min}$ & $86 \pm 20$ & $98 \pm 21$ & 0.052 \\
\hline
\end{tabular}

RSBI: Rapid shallow breathing index $\frac{\text { (Respiratory rate/min) }}{\text { Tidal volume }(\mathrm{L})}$,

$\mathrm{MV}_{\mathrm{e}}$ : Expired minute volume in $\mathrm{L} 1 \mathrm{~min}, \mathrm{P} / \mathrm{F}$ ratio: $\mathrm{PaO}_{2} / \mathrm{FiO}_{2}$

\begin{tabular}{lccc}
\hline \multicolumn{4}{l}{ Table 4: Respiratory outcomes after extubation } \\
\hline Parameter & Group I & Group II & $\boldsymbol{P}$ \\
\hline NIV, $n(\%)$ & $5(20)$ & $4(16)$ & 0.716 \\
Yes & $20(80)$ & $21(24)$ & \\
No & $53 \pm 36$ & $56 \pm 34$ & 0.279 \\
Duration of oxygen therapy (h) & $8 \pm 7$ & $6 \pm 5$ & 0.771 \\
LOICU stay (days) & & & \\
Mortality, $n(\%)$ & $2(20)$ & $2(20)$ & 1.0 \\
Yes & $23(80)$ & $23(80)$ & \\
No
\end{tabular}

NIV: Noninvasive ventilation, LOICU: Length of Intensive Care Unit

parameters were poorly predictive as weaning indices in a general ICU. ${ }^{[8]}$

The RSBI and RR were higher in the group weaned on T piece versus the group on PS in our groups of patients. The RSBI has been reported as the most accurate predictor of success of weaning in comparison to other respiratory parameters. ${ }^{[2,8,9]}$ Although the RSBI has been accepted as the most accurate test for weaning readiness in most studies, three other integrated indices of weaning, namely, the compliance, rate, oxygenation, and pressure index; compliance, oxygenation, rate, and effort index; and an integrated weaning index have been suggested as superior alternatives to assess weaning, and several studies have shown these to be superior to the RSBI in standard weaning protocols. ${ }^{[10]}$ The incorporation of these indices could have been more specific in evaluating weaning readiness for patients. In our study, the weaning parameter RSBI was compared in both the groups and RSBI was found to be significantly different between the groups; a higher value was recorded in T-piece patients which could be associated with increased work of breathing to overcome the resistance of artificial airway. Earlier studies have shown that T-piece trial can increase the work of breathing. ${ }^{[11]}$ Despite the fact that the patients receiving a T-piece trial had a graded weaning from PS to $\mathrm{T}$ piece before extubation, the $\mathrm{T}$ piece group showed a higher respiratory although it was $<22$ breaths/min and acceptable for weaning patients. It appears that $\mathrm{T}$ piece trial could evaluate the outcome of successful weaning even if the workload of breathing for the patient could be higher. A recent analysis of both modes for weaning has concluded that PS weaning is superior to T-piece trial for patients undergoing a simple weaning. ${ }^{[12]}$

Studies conducted do not show clear correlation between HR and RR changes associated with weaning. ${ }^{[13]}$ In our study, the $\mathrm{RR}$ was higher in the $\mathrm{T}$ piece group at all time points during weaning while the HR although higher at the start of weaning was not significantly different at $30 \mathrm{~min}$ and $60 \mathrm{~min}$ of the weaning trial.

Our secondary objective was to compare the need for respiratory assist devices after extubation, and this was not significantly different between the groups. However, reintubation rates were higher in the patients who required NIV, and these patients had a higher mortality also. A meta-analysis on 16 randomized controlled trials has shown that NIV reduced the reintubation rates and the occurrence of pneumonia postextubation in these patients. ${ }^{[14]}$

Duration of oxygen therapy, length of ICU stay and mortality rate were also compared in our study, and these were not significantly different between the two groups. Mortality between both groups was similar and was not related to respiratory events and included cardiac arrest, sepsis, and multiple organ failure.

Our group of patients was postsurgical patients without major respiratory comorbidity or infections, and this could explain a successful weaning in most patients included in our study.

Khamiees et al. ${ }^{[15]}$ stated that poor cough strength and increased secretions are important predictors of extubation outcomes. Salam et al. compared the effects of neurologic status, cough peak flow, and quantity of endotracheal secretions and 
Kumar, et al.: Extubation outcome - T-piece trial vs PSV

concluded that a decreased cough peak flow is a predictor of failed extubation in patients. ${ }^{[16]}$

The sedation protocols were uniform among our patients: Patients who underwent open abdominal surgery received an epidural analgesia during surgery and in the postoperative period while patients undergoing laparoscopic surgery received a three-tiered analgesic management. Both groups of patients received intravenous fentanyl for the duration of ventilation as neurosurgical patients. As per the ICU policy, sedation was discontinued in the morning and restarted when the patient was struggling or too awake. This interruption of sedation was developed from studies that documented the improved rates of weaning and shorter length of hospital stay in critically ill patients on mechanical ventilation. ${ }^{[17]}$ The groups of patients in our study had a shorter ventilatory time. We did not find any difficulty related to sedation levels in weaning.

\section{Conclusions}

Extubation outcomes are not different between weaning on T-piece versus PS. Weaning on T-piece is associated with increased RR, HR, RSBI, and lower oxygen saturation in comparison to PS group but does not affect extubation outcomes in postsurgical patients requiring mechanical ventilation for $>12 \mathrm{~h}$ before weaning.

\section{Financial support and sponsorship}

Nil.

\section{Conflicts of interest}

There are no conflicts of interest.

\section{RefEREnCeS}

1. Esteban A, Alía I, Gordo F, Fernández R, Solsona JF, Vallverdú I, et al. Extubation outcome after spontaneous breathing trials with T-tube or pressure support ventilation. The Spanish Lung Failure Collaborative Group. Am J Respir Crit Care Med 1997;156:459-65.

2. Alía I, Esteban A. Weaning from mechanical ventilation. Crit Care 2000;4:72-80.

3. Matić I, Majerić-Kogler V. Comparison of pressure support and T-tube weaning from mechanical ventilation: Randomized prospective study. Croat Med J 2004;45:162-6.
4. Esteban A, Alía I, Tobin MJ, Gil A, Gordo F, Vallverdú I, et al. Effect of spontaneous breathing trial duration on outcome of attempts to discontinue mechanical ventilation. Spanish Lung Failure Collaborative Group. Am J Respir Crit Care Med 1999;159:512-8.

5. Brochard L, Rauss A, Benito S, Conti G, Mancebo J, Rekik N, et al. Comparison of three methods of gradual withdrawal from ventilatory support during weaning from mechanical ventilation. Am J Respir Crit Care Med 1994;150:896-903.

6. Epstein SK, Ciubotaru RL. Independent effects of etiology of failure and time to reintubation on outcome for patients failing extubation. Am J Respir Crit Care Med 1998;158:489-93.

7. Haberthür C, Mols G, Elsasser S, Bingisser R, Stocker R, Guttmann J, et al. Extubation after breathing trials with automatic tube compensation, T-tube, or pressure support ventilation. Acta Anaesthesiol Scand 2002;46:973-9.

8. Conti G, Montini L, Pennisi MA, Cavaliere F, Arcangeli A, Bocci MG, et al. A prospective, blinded evaluation of indexes proposed to predict weaning from mechanical ventilation. Intensive Care Med 2004;30:830-6.

9. Yang KL, Tobin MJ. A prospective study of indexes predicting the outcome of trials of weaning from mechanical ventilation. N Engl J Med 1991;324:1445-50.

10. Tobin MJ, Jubran A. Variable performance of weaning-predictor tests: Role of Bayes' theorem and spectrum and test-referral bias. Intensive Care Med 2006;32:2002-12.

11. Haas CF, Loik PS. Ventilator discontinuation protocols. Respir Care 2012;57:1649-62.

12. Ladeira MT, Vital FM, Andriolo RB, Andriolo BN, Atallah AN, Peccin MS, et al. Pressure support versus T-tube for weaning from mechanical ventilation in adults. Cochrane Database Syst Rev 2014;2014;(5). Art No.:CD006056.

13. Papaioannou VE, Chouvarda I, Maglaveras N, Dragoumanis C, Pneumatikos I. Changes of heart and respiratory rate dynamics during weaning from mechanical ventilation: A study of physiologic complexity in surgical critically ill patients. J Crit Care 2011;26:262-72.

14. Glossop AJ, Shephard N, Bryden DC, Mills GH. Non-invasive ventilation for weaning, avoiding reintubation after extubation and in the postoperative period: A meta-analysis. Br J Anaesth 2012;109:305-14.

15. Khamiees M, Raju P, DeGirolamo A, Amoateng-Adjepong Y, Manthous CA. Predictors of extubation outcome in patients who have successfully completed a spontaneous breathing trial. Chest 2001;120:1262-70.

16. Salam A, Tilluckdharry L, Amoateng-Adjepong Y, Manthous CA. Neurologic status, cough, secretions and extubation outcomes. Intensive Care Med 2004;30:1334-9.

17. Girard TD, Kress JP, Fuchs BD, Thomason JW, Schweickert WD, Pun BT, et al. Efficacy and safety of a paired sedation and ventilator weaning protocol for mechanically ventilated patients in intensive care (Awakening and breathing controlled trial): A randomised controlled trial. Lancet 2008;371:126-34. 